\title{
Spectral synthesis for the differentiation operator in the Schwartz space
}

\author{
N.F. Abuzyarova 1
}

\begin{abstract}
We consider the spectral synthesis problem for the differentiation operator $D=\frac{\mathrm{d}}{\mathrm{d} t}$ in the Schwartz space $\mathcal{E}(a ; b)=C^{\infty}(a ; b)$ and the dual problem of local description for closed submodules in a special module of entire functions.
\end{abstract}

\section{Introduction}

Let $(a ; b)$ be a finite or infinite open interval of the real line and let

$$
\left[a_{1} ; b_{1}\right] \Subset\left[a_{2} ; b_{2}\right] \Subset \ldots,
$$

be a sequence of segments exhausting this interval. We consider the Schwartz space $\mathcal{E}(a ; b)=C^{\infty}(a ; b)$, equipped by the metrizible topology of projective limit of the Banach spaces $C^{k}\left[a_{k} ; b_{k}\right]$. It is known that $\mathcal{E}(a ; b)$ is a reflexive Fréchet space. Unless otherwise specified, we denote by $W$ a closed nontrivial subspace of $\mathcal{E}(a ; b)$ which is invariant under the differentiation operator Shortly, we say such a subspace $W$ to be $D$-invariant.

Let $\operatorname{Exp} W$ denote the set of all exponential monomials $t^{j} e^{-\mathrm{i} \lambda t}$, that is, the set of all root elements the operator $D$ contained in $W$. As we will see later, the set $\operatorname{Exp} W$ is at most countable.

A generalization of L. Euler fundamental principle for finite order differential equations with constant coefficients [1], as well as a generalization of classical results on mean periodic functions due to L.Schwartz [2], is the spectral synthesis for a $D$-invariant subspace $W$ :

$W=\overline{\mathcal{L}(\operatorname{Exp} W)}, \quad$ where $\quad \mathcal{L}(\cdot)$ denotes the linear span of the set $(\cdot)$.

\footnotetext{
${ }^{1}$ This work was supported by the Ministry of Education and Science of Russian Federation, project no. 01201456408 .
} 
A. Aleman and B. Korenblum notice in [3] that there exist non-trivial $D$-invariant subspaces $W$ of $\mathcal{E}(a ; b)$ with the property $\operatorname{Exp} W=\emptyset$. Namely, let $I \subset(a ; b)$ be a non-empty relatively closed interval and set

$$
W_{I}=\left\{f \in \mathcal{E}: \quad f^{(k)}(t)=0, t \in I, k=0,1,2, \ldots\right\} .
$$

Obviously, the subspace $W_{I}$ is $D$-invariant and the set $\operatorname{Exp} W_{I}$ is empty. In what follows, $W_{I}$ doesn't admit the spectral synthesis (1.1).

For a $D$-invariant subspace $W$, we denote by $I_{W}$ a relatively closed in $(a ; b)$ interval which is minimal among all intervals $I$ with the property $W_{I} \subset W$. The existence of $I_{W}$ follows from [3, Theorem 4.1].

In [3], its authors propose a weaker, than (1.1), version of spectral synthesis for the differentiation operator $D$ in the space $\mathcal{E}(a ; b)$. We call it spectral synthesis in the weak sense (or, weak spectral synthesis). This version takes into account the presence of the residual subspace $W_{I_{W}} \subset W . D$-invariant subspace $W$ is said to be admitting spectral synthesis in the weak sense (or, admitting weak spectral synthesis) if

$$
W=\overline{W_{I_{W}}+\mathcal{L}(\operatorname{Exp} W)}
$$

The authors of the paper [3] prove that the spectrum $\sigma(W)$ of $D$-invariant subspace $W$, which is defined to be the spectrum of the restriction of the operator $D$ to $W$, is either discrete or equal to the whole complex plane ([3, Theorem 2.1]). In the first case, the spectrum $\sigma(W)$ is a sequence of multiple points

$$
\sigma(W)=\left\{\left(-\mathrm{i} \lambda_{j}, m_{j}\right): \lambda_{j} \in \mathbb{C}, \quad m_{j} \in \mathbb{N}, j=1,2, \ldots\right\},
$$

and $\operatorname{Exp} W=\left\{t^{k} e^{-\mathrm{i} \lambda_{j} t}: \quad k=0,1, \ldots, m_{j}-1, j \in \mathbb{N}\right\}$.

If the subspace $W$ has the form (1.3) , its spectrum is discrete and equals $(-\mathrm{i} \Lambda)$, where $\Lambda=\left\{\left(\lambda_{j}, m_{j}\right)\right\}$. Such a subspace admits spectral synthesis (1.1) under the additional assumption $I_{W}=(a ; b)$.

A. Aleman and B. Korenblum conjecture in [3, Section 6] that any $D$ invariant subspace $W \subset \mathcal{E}(a ; b)$ with discrete spectrum has the form (1.3). They prove this conjecture for the case of a subspace with finite (in particular, empty) spectrum [3, Propositions 4.1, 6.1]

In [4] this conjecture is studied by developing and improving methods of [3] and reducing the weak spectral synthesis problem to the problem of completeness for mixed systems in a Hilbert space. 
To formulate the main result of the paper [4] we recall that the completeness radius $\rho_{\Lambda}$ of a sequence $\Lambda=\left\{\left(\lambda_{j}, m_{j}\right)\right\}$ is defined as the infimum of the radii of (open) intervals $I \subset \mathbb{R}$ for which the system of exponential monomials $\left\{t^{k} e^{-\mathrm{i} \lambda_{j} t}, \quad k=0, \ldots, m_{j}-1, \quad j=1,2, \ldots\right\}$ is not complete in the spaces $\mathcal{E}(I), C(I), L^{p}(I), 1 \leq p<\infty$ (see [5]).

Theorem 1.1 [4] positively answers the question on the weak spectral synthesis for $D$-invariant subspace $W \subset \mathcal{E}(a ; b)$, with discrete spectrum of the form (1.4) if $2 \rho_{\Lambda}<\left|I_{W}\right|$, where $\Lambda=\mathrm{i} \sigma(W)$ and $\left|I_{W}\right|$ is the length of the interval $I_{W}$. It turns out that the relation (1.3) may fail for $D$-invariant subspaces $W$ with discrete spectrum $\sigma(W)=-\mathrm{i} \Lambda$ if $2 \rho_{\Lambda}=\left|I_{W}\right|$. First example of such a subspace is constructed in [4, Theorem 1.2]. A lot of examples, including the mentioned one, arise from [6, Theorem 3]. In the other hand, as our example in Section 3 below shows, $D$-invariant subspace $W$ with discrete spectrum $\sigma(W)=-\mathrm{i} \Lambda$ satisfyng the relation $2 \rho_{\Lambda}=\left|I_{W}\right|$, may also be of the form (1.3).

We announce in [7] the necessary and sufficient condition for $D$-invariant subspace $W \subset \mathcal{E}(a ; b)$ to be admitting weak spectral synthesis. Our approach to the problem does completely differ from the methods used in the works [3], 4. Namely, we apply a dual scheme going back to I.F Krasichkov-Tenovskii [8] and L. Ehrenpreis [9]. This scheme reduces the problems concerning $D$-invariant subspaces of the space $\mathcal{E}(a ; b)$ to the equivalent ones on closed submodules in a special weighted module of entire functions. We formulate the mentioned criterion in terms of the annihilator submodule of $D$-invariant subspace $W$ [7, Theorem 3]. Then, we deduce from it different sufficient conditions of admitting of weak spectral synthesis by $D$-invariant subspace $W$ with discrete spectrum ([7, Corollaries 1 and 2], Theorem [6 below, [4, Theorems 1.1, 1.3]).

This paper contains the explicit proofs for most of the assertions announced in [7] concerning weak spectral synthesis in the space $\mathcal{E}(a ; b)$. In addition, we prove one theorem on weak spectral synthesis for "translationinvariant" subspaces of $\mathcal{E}(a ; b)$ (Theorem 6 below).

The organization of the paper is as follows. In Section 2 we describe the mentioned dual scheme. Section 3 contains a series of results on submodules of entire functions. At last, in Section 4 we derive assertions on $D$-invariant subspaces in $\mathcal{E}(a ; b)$. 


\section{Dual scheme}

1. Let us define a locally convex space of entire functions $\mathcal{P}(a ; b)$ to be equal to inductive limit of the sequence of the Banach spaces $\left\{P_{k}\right\}$, where $P_{k}$ consists of all entire functions $\varphi$ with finite norm

$$
\|\varphi\|_{k}=\sup _{z \in \mathbb{C}} \frac{|\varphi(z)|}{(1+|z|)^{k} \exp \left(b_{k} y^{+}-a_{k} y^{-}\right)}, \quad y^{ \pm}=\max \{0, \pm y\}, \quad z=x+\mathrm{i} y .
$$

The inclusions $P_{k} \subset P_{k+1}$ are compact for each $k=1,2, \ldots$ It follows that the space $\mathcal{P}(a ; b)$ is a locally convex space of $\left(L N^{*}\right)$-type (see [10]). Further, the operation of multiplication by an independent variable $z$ acts continuously in this space, that is, $\mathcal{P}(a ; b)$ is a topological module over the polynomial ring $\mathbb{C}[z]$.

It is well-known that the Fourier-Laplace transform $\mathcal{F}$ establishes a linear topological isomorphism between the strong dual space $\mathcal{E}^{\prime}(a ; b)$ and the space $\mathcal{P}(a ; b)$ [11, chapter 7, theorem 7.3.1]:

$$
S \in \mathcal{E}^{\prime}(a ; b) \longleftrightarrow \varphi \in \mathcal{P}(a ; b) \Longleftrightarrow \varphi=\mathcal{F}(S)=\left(S, e^{-\mathrm{i} t z}\right)
$$

Let $\mathcal{J} \subset \mathcal{P}(a ; b)$ be a closed submodule, that is, $\mathcal{J}$ is a closed subspace with the property $z \mathcal{J} \subset \mathcal{J}$. Two natural characteristics of the submodule $\mathcal{J}$ arise immediately: its zero set $\Lambda_{\mathcal{J}}$ and its indicator segment $\left[c_{\mathcal{J}} ; d_{\mathcal{J}}\right]$. The first one, $\Lambda_{\mathcal{J}}$, is well-known. We always meet it when dealing with the local description problem for ideals and submodules of holomorphic functions. The notion of the indicator segment is introduced in [7], [13. Below we recall both definitions. Given a function $\varphi \in \mathcal{P}(a ; b)$, we set at any $\lambda \in \mathbb{C}$

$$
n_{\varphi}(\lambda)=\left\{\begin{array}{l}
0, \quad \text { if } \varphi(\lambda) \neq 0 \\
m, \quad \text { if } \lambda \text { is a zero of } \varphi \text { of muliplicity } m
\end{array}\right.
$$

The divisor of a closed submodule $\mathcal{J} \subset \mathcal{P}(a ; b)$ is defined as $n_{\mathcal{J}}(\lambda)=\min _{\varphi \in \mathcal{J}} n_{\varphi}(\lambda)$.

The zero set of a non-zero function $\varphi \in \mathcal{P}(a ; b)$ is

$$
\Lambda_{\varphi}=\left\{\left(\lambda_{k}, m_{k}\right): m_{k}=n_{\varphi}\left(\lambda_{k}\right)>0, k=1,2, \ldots\right\}
$$

The zero set of a submodule $\mathcal{J} \neq\{0\}$ is

$$
\Lambda_{\mathcal{J}}=\left\{\left(\lambda_{k}, m_{k}\right): m_{k}=n_{\mathcal{J}}\left(\lambda_{k}\right)>0, k=1,2, \ldots\right\}
$$


As it is known (see, e.g., [12]), any element of the space $\mathcal{P}(a ; b)$ is a function of completely regular growth with respect to the order 1 , and the indicator diagram of any $\varphi \in \mathcal{P}(a ; b)$ is a segment $\left[\mathrm{i} c_{\varphi} ; \mathrm{i} d_{\varphi}\right] \subset(\mathrm{i} a ; \mathrm{i} b)$. The indicator segment $\left[c_{\mathcal{J}} ; d_{\mathcal{J}}\right]$ of a submodule $\mathcal{J}$ is defined to be the segment (generally speaking, in $\overline{\mathbb{R}}$ ) with the endpoints

$$
c_{\mathcal{J}}=\inf _{\varphi \in \mathcal{J}} c_{\varphi}, \quad d_{\mathcal{J}}=\sup _{\varphi \in \mathcal{J}} d_{\varphi}
$$

For a closed subspace $W \subset \mathcal{E}(a ; b)$ we set

$$
W^{0}=\left\{S \in \mathcal{E}^{\prime}(a ; b): \quad(S, f)=0, \quad f \in W\right\} .
$$

This is a closed subspace of the strong dual space $\mathcal{E}^{\prime}(a ; b)$. It is said to be the annihilator subspace of $W$.

The following proposition establishes the duality between $D$-invariant subspaces $W \subset \mathcal{E}(a ; b)$ and closed submodules $\mathcal{J} \subset \mathcal{P}(a ; b)$ [13, proposition $1]$.

Proposition 1 (Duality principle.) Between the set $\{W\}$ of all D-invariant subspaces of the space $\mathcal{E}(a ; b)$ and the set $\{\mathcal{J}\}$ of all closed submodules of the module $\mathcal{P}(a ; b)$, there is a one-to-one correspondence, namely,

$$
W \longleftrightarrow \mathcal{J} \Longleftrightarrow \mathcal{J}=\mathcal{F}\left(W^{0}\right)
$$

Moreover,

$$
\operatorname{Exp} W=\left\{t^{j} e^{-i \lambda_{k} t}: j=0, \ldots m_{k}-1, \quad\left(\lambda_{k}, m_{k}\right) \in \Lambda_{\mathcal{J}}\right\}
$$

and the endpoints of the interval $I_{W}$ are $c_{\mathcal{J}}$ and $d_{\mathcal{J}}$.

Obviously, $D$-invariant subspace $W$ admits weak spectral synthesis if and only if it is the minimal one among all $D$-invariant subspaces $\tilde{W}$ with properties

$$
I_{\tilde{W}}=I_{W}, \quad \operatorname{Exp} \tilde{W}=\operatorname{Exp} W .
$$

By the duality principle (proposition 1), we see that the annihilator submodule $\mathcal{J}=\mathcal{F}\left(W^{0}\right)$ of such a subspace $W$ should be maximal one among all submodules $\tilde{\mathcal{J}} \subset \mathcal{P}(a ; b)$ such that

$$
\Lambda_{\tilde{\mathcal{J}}}=\Lambda_{\mathcal{J}}, \quad\left[c_{\tilde{\mathcal{J}}} ; d_{\tilde{\mathcal{J}}}\right]=\left[c_{\mathcal{J}} ; d_{\mathcal{J}}\right]
$$


If so, we say the submodule $\mathcal{J}$ to be weakly loclizable. In the other words, submodule $\mathcal{J} \subset \mathcal{P}(a ; b)$ is weakly localizable if it contains any function $\psi \in$ $\mathcal{P}(a ; b)$, which vanishes (with the multiplicities) on the set $\Lambda_{\mathcal{J}}$ and has the indicator diagram $\left[\mathrm{i} c_{\psi} ; \mathrm{i} d_{\psi}\right] \subset\left[\mathrm{i} c_{; J} ; \mathrm{i} d_{\mathcal{J}}\right]$.

As a conclusion we get

Proposition 2 D-invariant subspace $W \subset \mathcal{E}(a ; b)$ admits weak spectral synthesis if and only if its annihilator submodule $\mathcal{J}=\mathcal{F}\left(W^{0}\right) \subset \mathcal{P}(a ; b)$ is weakly localizable.

Let us notice that the weak localizable submodule $\mathcal{J}=\mathcal{F}\left(W^{0}\right)$ turns to be localizable or ample (see [14], [15]) if

$$
I_{W}=\left[c_{\mathcal{J}} ; d_{\mathcal{J}}\right] \bigcap(a ; b)=(a ; b)
$$

Under the assumption 2.3 , Proposition 2 becomes the classical duality principle (cf. [8]).

Unless otherwise specified, we consider only closed submodules $\mathcal{J} \subset$ $\mathcal{P}(a ; b)$.

A submodule $\mathcal{J}$ is said to be stable at a point $\lambda \in \mathbb{C}$ if for any $\varphi \in \mathcal{J}$ the inequality $n_{\varphi}(\lambda)>n_{\mathcal{J}}(\lambda)$ implies $\varphi /(z-\lambda) \in \mathcal{J}$. A submodule $\mathcal{J}$ is said to be stable if it is stable at any point $\lambda \in \mathbb{C}$. These notions are introduced in [8], [14].

The propositions [4, Proposition 3.1] and [13, Proposition 2], taking together, can be expressed as the following assertion.

Proposition 3 The spectrum of D-invariant subspace $W \subset \mathcal{E}(a ; b)$ is discrete if and only if its annihilator submodule $\mathcal{J}$ is stable.

\section{Weakly localizable submodules in $\mathcal{P}(a ; b)$.}

1. Proposition 2 reduces the problem of weak spectral synthesis for $D$ invariant subspace $W$ to the studying of weak localizability of its annihilator submodule $\mathcal{J}$. One can easy see that a weakly localizable submodule is necessarily stable.

Below we recall the definitions of some notions we will need in the further considerations. These notions have been introduced in [14, $\S 1$ and $\S 3$, remark 2 ]. We give them for the case of scalar functions. 
A submodule $\mathcal{J}$ is $b$-saturated with respect to a function $\psi \in \mathcal{P}(a ; b)$ if there exists a bounded set $B \subset \mathcal{P}(a ; b)$ sucn that the following implication is true: if $\Phi(z)$ is an entire function and the inequality $|\Phi(z) \varphi(z)| \leq|\varphi(z)|+$ $|\psi(z)|$ holds each $z \in \mathbb{C}$ and for any function $\varphi \in B \bigcap \mathcal{J}$, then $\Phi=$ const.

A locally convex space $P$ of entire functions is said to be $b$-stable if, for any bounded set $B \subset \mathcal{P}$, the set of all entire functions $\psi$ of the form

$$
\psi(z)=\varphi(z) /(z-\lambda), \quad \lambda \in \mathbb{C}, \quad \varphi \in B,
$$

is bounded in $P$.

As we have noticed above, $\mathcal{P}(a ; b)$ is a $\left(L N^{*}\right)$-type space. It follows that a subset $B \subset \mathcal{P}(a ; b)$ is bounded if and only if, for some $k$, it is contained and bounded in the Banach space $P_{k}[10$, Theorem 2]. Using this fact and the definition of the topology in $\mathcal{P}(a ; b)$ we can easily verify that the space $\mathcal{P}(a ; b)$ is a bornologic and $b$-stable. For spaces of holomorphic vector-functions, which are bornologic and $b$-stable, I.F. Krasichkov-Ternovskii proved the following theorem [14]. (We cite it here for the case of our space $\mathcal{P}(a ; b)$.)

Bornological Version of Individual Theorem. Let $\mathcal{J}$ be a stable submodule in $\mathcal{P}(a ; b)$, and let $\psi \in \mathcal{P}(a ; b)$ be a function satisfying the following assumption: $n_{\psi}(z) \geq n_{\mathcal{J}}(z)$ in the whole complex plane. Then, $\psi \in \mathcal{J}$ if and only if $\mathcal{J}$ is b-saturated with respect to $\psi$.

This assertion allows us to obtain the criterion of weak localizibilty for closed submodules of the module $\mathcal{P}(a ; b)$.

Given a function $\varphi \in \mathcal{P}(a ; b)$, we denote by $\mathcal{J}(\varphi)$ the submodule formed by all functions $\psi=\omega \varphi \in \mathcal{P}(a ; b)$. Here $\omega$ is an entire function of minimal exponential type with the integral $\int_{-\infty}^{\infty}\left(\log ^{+}|\omega(x)| /\left(1+x^{2}\right)\right) \mathrm{d} x$ to be converging. In the other words, $\mathcal{J}(\varphi)$ consists of all functions $\psi \in \mathcal{P}(a ; b)$, which are dividing by $\varphi$ and have the same indicator diagram as $\varphi$. Clearly, the submodule $\mathcal{J}(\varphi)$ is weakly localizable.

To begin with, we prove one auxiliary assertion.

Lemma 1 Let $\mathcal{J}$ be a stable submodule. Suppose that for a function $\varphi \in$ $\mathcal{P}(a ; b)$ the inequality $n_{\varphi}(z) \geq n_{\mathcal{J}}(z)$ holds for any $z \in \mathbb{C}$, and its indicator diagram $\left[i c_{\varphi} ; i d_{\varphi}\right]$ is a subset of the open interval $\left(i c_{\mathcal{J}} ; i d_{\mathcal{J}}\right)$.

Then $J(\varphi) \subset \mathcal{J}$.

Proof. Let $\tilde{\varphi}$ be an arbitrary function in $\mathcal{J}(\varphi)$. By assumption, we have $c_{\mathcal{J}}<c_{\varphi}$. Using the definition of $c_{\mathcal{J}}$ we can find a function $\varphi_{1} \in \mathcal{J}$ with 
the property

$$
c_{\mathcal{J}} \leq c_{\varphi_{1}}<c_{\varphi}
$$

We can also find another function $\varphi_{2} \in \mathcal{J}$ satisfying the inequalities

$$
d_{\varphi}<d_{\varphi_{1}} \leq d_{\mathcal{J}}
$$

Set $\varphi_{B}=\varphi_{1}+\varphi_{2}$. It follows from the definition of the submodule $\mathcal{J}(\varphi)$ that the indicator diagram of an arbitrary function $\tilde{\varphi} \in \mathcal{J}(\varphi)$ is the segment $\left[\mathrm{i} c_{\varphi} ; \mathrm{i} d_{\varphi}\right]$. Therefore, this indicator diagram is a compact subset of the indicator diagram $\left[\mathrm{i} c_{\varphi_{B}} ; \mathrm{i} d_{\varphi_{B}}\right]$. Taking into the account that the function $\varphi_{B}$ has completely regular growth, we get

$$
\frac{\tilde{\varphi}(z)}{\varphi_{B}(z)} \rightarrow 0
$$

where $z=r e^{i \theta}$ and $r$ tends to $\infty$ lying outside a set of zero relative measure. Moreover, the relation (3.1) holds uniformly on $\theta \in\{|\pi / 2-\theta|<\delta\} \bigcup\{\mid-$ $\pi / 2-\theta \mid<\delta\}$ if $\delta>0$ is sufficiently small.

Now, it is not difficult to show that the submodule $\mathcal{J}$ is $b$-saturated with respect to any function $\tilde{\varphi} \in \mathcal{J}(\varphi)$. For this purpose we set $B=\left\{\varphi_{B}\right\}$ and consider an arbitrary entire function $\omega$ satisfying the inequality

$$
\left|\omega(z) \varphi_{B}(z)\right| \leq|\tilde{\varphi}(z)|+\left|\varphi_{B}(z)\right|, \quad z \in \mathbb{C} .
$$

From the relations (3.1), (3.2), by the maximum modulus principle, we derive that the function $\omega$ is bounded on the imaginary axis. The relation (3.2) and the completely regular growth of the function $\varphi_{B}$ imply that the function $\omega$ has minimal exponential type. In what follows, $\omega=$ const and the stable submodule $\mathcal{J}$ is $b$-saturated with respect to the function $\tilde{\varphi}$. Applying the above cited Bornological Version of Individual Theorem, we conclude that $\tilde{\varphi} \in \mathcal{J}$.

Theorem 1 Assume that $\mathcal{J}$ is a stable submodule. This submodule is weakly localizable if and only if there exists a function $\varphi \in \mathcal{J}$ with the property

$$
\mathcal{J}(\varphi) \subset \mathcal{J}
$$


Proof. We should notice that the assertion is trivial when $c_{\mathcal{J}}=d_{\mathcal{J}}$. Indeed, in this case $\mathcal{J}$ is exactly the set $\left\{\alpha e^{-\mathrm{i} c_{\mathcal{J}} z}\right\}, \alpha \in \mathbb{C}$. In what follows, we assume that $c_{\mathcal{J}}<d_{\mathcal{J}}$.

Clearly, we only need to prove the sufficient part of the theorem: the existence of a function $\varphi \in \mathcal{J}$ with the property (3.3) implies that the submodule $\mathcal{J}$ is weakly localizable.

1) First, we suppose that (3.3) holds for a function $\varphi$ which indicator diagram coincides with to the segment $\left[\mathrm{i} c_{\mathcal{J}} ; \mathrm{i} d_{\mathcal{J}}\right]$.

Given an arbitrary function $\psi \in \mathcal{P}(a ; b)$ with zero set $\Lambda_{\psi} \supset \Lambda_{\mathcal{J}}$ and indicator diagram contained in $\left[\mathrm{i} c_{\mathcal{J}} ; \mathrm{i} d_{\mathcal{J}}\right]$, we will verify that the submodule $\mathcal{J}$ is $b$-saturated with respect to the function $\psi$. Set

$$
B=\{\tilde{\varphi} \in \operatorname{Hol}(\mathbb{C}): \quad|\tilde{\varphi}(z)| \leq|\psi(z)|+|\varphi(z)|, \quad z \in \mathbb{C}\} .
$$

From the topological properties of the space $\mathcal{P}(a ; b)$, it follows that $B$ is a bounded subset of $\mathcal{P}(a ; b)$. Let $\omega$ be an entire function satisfying the inequality

$$
|\omega(z) \tilde{\varphi}(z)| \leq|\tilde{\varphi}(z)|+|\psi(z)|
$$

in the whole complex plane for any function $\tilde{\varphi} \in B \cap \mathcal{J}$. In particular, this inequality is true when $\tilde{\varphi}=\varphi$. In what follows, $\omega$ is a function of minimal exponential type and $\omega \varphi \in \mathcal{J}(\varphi)$. The inclusion $\mathcal{J}(\varphi) \subset \mathcal{J}$, (3.4) and the definition of the set $B$, lead to the inclusion $\omega \varphi \in B \cap \mathcal{J}$. Setting $\tilde{\varphi}=\omega \varphi$ in (3.4), we get the inequalities

$$
\left|\omega^{2}(z) \varphi(z)\right| \leq|\omega(z) \varphi(z)|+|\psi(z)| \leq 2(|\varphi(z)|+|\psi(z)|),
$$

for any $z \in \mathbb{C}$. Further, arguing as we have done for the function $\omega \varphi$, we derive that

$$
\frac{\omega^{2}}{2} \varphi \in B \bigcap \mathcal{J} .
$$

Now, setting $\tilde{\varphi}=\frac{\omega^{2}}{2} \varphi$ in (3.4) gives us the relations

$$
\left|\frac{\omega^{3}(z)}{2} \varphi(z)\right| \leq\left|\frac{\omega^{2}(z)}{2} \varphi(z)\right|+|\psi(z)| \leq 2(|\varphi(z)|+|\psi(z)|)
$$

in the whole complex plane. In what follows, the inclusion

$$
\frac{\omega^{3}}{2^{2}} \varphi \in B \bigcap \mathcal{J}
$$


is valid.

Continuing to argue by the similar way, we obtain that

$$
\frac{\left|\omega^{n}(z)\right|}{2^{n-1}}|\varphi(z)| \leq|\varphi(z)|+|\psi(z)|
$$

for each $n \in \mathbb{N}$ and for any $z \in \mathbb{C}$. The only case when it can happen is $\omega=$ const. Hence, the submodule $\mathcal{J}$ is $b$-saturated with respect to the function $\psi$. Applying Bornological Version of Individual Theorem we get the inclusion $\psi \in \mathcal{J}$. This is true for any function $\psi \in \mathcal{P}(a ; b)$ satisfying $\Lambda_{\psi} \supset \Lambda_{\mathcal{J}}$. Therefore, we conclude that the submodule $\mathcal{J}$ is weakly localizable.

2) Let us now consider the case when $\mathcal{J}(\varphi) \subset \mathcal{J}$ and the indicator dia$\operatorname{gram}\left[\mathrm{i} c_{\varphi} ; \mathrm{i} d_{\varphi}\right]$ of the function $\varphi$ is a proper subset of the segment $\left[\mathrm{i} c_{\mathcal{J}} ; \mathrm{i} d_{\mathcal{J}}\right]$. We also assume in this part of the proof that $\left[\mathrm{i} c_{\mathcal{J}} ; \mathrm{i} d_{\mathcal{J}}\right] \subset(\mathrm{i} a ; \mathrm{i} b)$. The restriction on the indicator diagram $\left[\mathrm{i} c_{\varphi} ; \mathrm{i} d_{\varphi}\right]$ implies that at least one of the values $\delta_{1}=c_{\varphi}-c_{\mathcal{J}} \geq 0, \delta_{2}=d_{\mathcal{J}}-d_{\varphi} \geq 0$ is strictly positive. Suppose, e.g., that $\delta_{1}>0$ and $\delta_{2}>0$. Then, by Lemma 1, we get $\mathcal{J}\left(e^{i \delta^{\prime} z} \varphi\right) \subset \mathcal{J}$ for any $\delta^{\prime} \in\left[0 ; \delta_{1}\right)$ and $\mathcal{J}\left(e^{-i \delta^{\prime \prime} z} \varphi\right)$, for any $\delta^{\prime \prime} \in\left[0 ; \delta_{2}\right)$. In particular,

$$
e^{i \delta^{\prime} z} \varphi, \quad e^{-i \delta^{\prime \prime} z} \varphi \in \mathcal{J} \quad \text { for any } \quad \delta^{\prime} \in\left[0 ; \delta_{1}\right), \delta^{\prime \prime} \in\left[0 ; \delta_{2}\right) .
$$

Note that the relations

$$
\lim _{\delta^{\prime} \rightarrow \delta_{1}} e^{i \delta^{\prime} z} \varphi=e^{i \delta_{1} z} \varphi, \quad \lim _{\delta^{\prime \prime} \rightarrow \delta_{2}} e^{i \delta^{\prime \prime} z} \varphi=e^{i \delta_{2} z} \varphi
$$

are valid in the topology of $\mathcal{P}(a ; b)$. These relations, together with (3.6), lead to the inclusion $\Phi \in \mathcal{J}$, where $\Phi=\left(e^{i \delta_{1} z}+e^{-i \delta_{2} z}\right) \varphi$. Further, any function $\Psi \in \mathcal{J}(\Phi)$ has the form

$$
\Psi=\omega \Phi=\omega\left(e^{i \delta_{1} z}+e^{-i \delta_{2} z}\right) \varphi
$$

where $\omega$ is an entire function of minimal exponential type. There is no difficulty to verify that $\omega \varphi \in \mathcal{P}(a ; b)$, and Lemma 1 implies the inclusions

$$
\omega \varphi \in \mathcal{J}, \quad e^{i \delta^{\prime} z} \omega \varphi \in \mathcal{J}, \quad e^{-i \delta^{\prime \prime} z} \omega \varphi \in \mathcal{J}
$$

for each $\delta^{\prime} \in\left(0 ; \delta_{1}\right)$ and for each $\delta^{\prime \prime} \in\left(0 ; \delta_{2}\right)$. We can easily see that

$$
e^{i \delta^{\prime} z} \omega \varphi+e^{-i \delta^{\prime \prime} z} \omega \varphi \rightarrow \Psi \quad \text { as } \quad \delta^{\prime} \rightarrow \delta_{1}, \quad \delta^{\prime \prime} \rightarrow \delta_{2}
$$


in the topology of $\mathcal{P}(a ; b)$, and, consequently, $\Psi \in \mathcal{J}$. Finally, we obtain the inclusion $\mathcal{J}(\Phi) \subset \mathcal{J}$.

The indicator diagram of the function $\Phi$ is equal to the segment $\left[i c_{\mathcal{J}} ; \mathrm{i} d_{\mathcal{J}}\right]$. By this observation and the first part of the proof, we conclude that the submodule $\mathcal{J}$ is weakly localizable.

If one of the values $\delta_{1}$ or $\delta_{2}$ equals zero we should argue by the similar way, with obvious changes.

3) It remains to consider the case when at least one of the endpoints of the indicator segment $\left[c_{\mathcal{J}} ; d_{\mathcal{J}}\right]$ coincides with the corresponding endpoint of the interval $(a ; b)$ (that is, $c_{\mathcal{J}}=a$ or $d_{\mathcal{J}}=b$ ).

Given any function $\Psi \in \mathcal{P}(a ; b)$ with the indicator diagram $\left[\mathrm{i} c_{\Psi} ; \mathrm{i} d_{\Psi}\right] \subset$ $\left[\mathrm{i} c_{\mathcal{J}} ; \mathrm{i} d_{\mathcal{J}}\right]$ and the zero set $\Lambda_{\Psi} \supset \Lambda_{\mathcal{J}}$, we should prove the inclusion $\Psi \in \mathcal{J}$. Let $\left[c^{\prime} ; d^{\prime}\right]$ be a segment subjected the following restrictions:

$$
\left[c^{\prime} ; d^{\prime}\right] \subset(a ; b) \bigcap\left[c_{\mathcal{J}} ; d_{\mathcal{J}}\right], \quad\left[c_{\Psi} ; d_{\Psi}\right] \subset\left[c^{\prime} ; d^{\prime}\right], \quad\left[c_{\varphi} ; d_{\varphi}\right] \subset\left[c^{\prime} ; d^{\prime}\right] .
$$

We denote by $\mathcal{J}^{\prime}$ a weakly localizable submodule with the zero set $\Lambda_{\mathcal{J}^{\prime}}=\Lambda_{\mathcal{J}}$ and the indicator segment $\left[c^{\prime} ; d^{\prime}\right]$. Then, $\tilde{\mathcal{J}}=\mathcal{J} \cap \mathcal{J}^{\prime}$ is a stable submodule with the zero set $\Lambda_{\tilde{\mathcal{J}}}=\Lambda_{\mathcal{J}}$ and the indicator segment $\left[c^{\prime} ; d^{\prime}\right]$.

The relations (3.7) lead to the inclusion $\mathcal{J}(\varphi) \subset \tilde{\mathcal{J}}$. Therefore, by the previous parts of the proof, we get that $\tilde{\mathcal{J}}=\mathcal{J}^{\prime}$. Taking into account the relations (3.7) one more time, we conclude that $\Psi \in \tilde{\mathcal{J}} \subset \mathcal{J}$.

Remark 1 In the first part of the proof we use the argument which is similar to the one has been used by I.F. Krasichkov-Ternovskii [16, §11, Theorem 11.1], [15, §5, Proposition 5.5].

Given a function $\varphi \in \mathcal{P}(a ; b)$, we, as usual, define the principal submodule generated by $\varphi$ to be the closure of the set $\{p \varphi: p \in \mathbb{C}[z]\}$ in $\mathcal{P}(a ; b)$. We denote this submodule by $\mathcal{J}_{\varphi}$.

Theorem 2 Let $\mathcal{J}$ be a stable submodule. Each of the assumptions listed below is sufficient for the submodule $\mathcal{J}$ to be weakly localizable:

1) there exists a function $\varphi \in \mathcal{J}$ which generates the weakly localizable principal submodule $\mathcal{J}_{\varphi}$;

2) the inequality

$$
2 \rho_{\Lambda_{\mathcal{J}}}<d_{\mathcal{J}}-c_{\mathcal{J}}
$$

holds, where $d_{\mathcal{J}}-c_{\mathcal{J}}=+\infty$ if at least one of the values $c_{\mathcal{J}}$ or $d_{\mathcal{J}}$ is not finite. 
Proof. 1) Let $\varphi \in \mathcal{J}$ be a function generating the weakly localizable principal submodule $\mathcal{J}_{\varphi}$. Then, $\mathcal{J}_{\varphi}=\mathcal{J}(\varphi) \subset \mathcal{J}$, and Theorem 1 implies that the submodule $\mathcal{J}$ is weakly localizable.

2) Suppose that the inequality (3.8) holds. According to the definition of $\rho_{\Lambda}$ and [11, chapter 7, Theorem 7.3.1], there exists a function $\varphi \in \mathcal{P}(a ; b)$ with the zero set $\Lambda_{\varphi} \supset \Lambda_{\mathcal{J}}$ and the indicator diagram $\left[\mathrm{i} c_{\varphi} ; \mathrm{i} d_{\varphi}\right] \subset\left(\mathrm{i} c_{\mathcal{J}} ; \mathrm{i} d_{\mathcal{J}}\right)$. By Lemma 1 we get the inclusion $\mathcal{J}(\varphi) \subset \mathcal{J}$. Applying Theorem 1 we conclude that the submodule $\mathcal{J}$ is weakly localizable.

Corollary 1 A stable submodule $\mathcal{J}$ is ample (localizable) if and only if its indicator segment is equal to the segment $[a ; b] \subset[-\infty ;+\infty]$.

Remark 2 To study the weak localizability of stable submodules by verifying the assumption 1) of Theorem 2 is only reasonable when $2 \rho_{\Lambda_{\mathcal{J}}}=d_{\mathcal{J}}-c_{\mathcal{J}}$. If so, we have $\left[c_{\mathcal{J}} ; d_{\mathcal{J}}\right] \subset(a ; b)$, and the indicator diagram of any function $\psi \in \mathcal{J}$ is equal to $\left[\mathrm{i} c_{\mathcal{J}} ; \mathrm{i} d_{\mathcal{J}}\right]$.

A function $\varphi \in \mathcal{P}(-\infty ;+\infty)$ is said to be invertible (see [17]) if for any $\Phi \in \mathcal{P}(-\infty ;+\infty)$ the function $\Phi / \varphi$ belongs to $\mathcal{P}(-\infty ;+\infty)$ whenever it is an entire one.

The assumption 1) of Theorem 2 is valid if the submodule $\mathcal{J}$ contains an invertible function or, more generally, a function $\varphi$ satisfying the relations

$$
\mathcal{J}_{\varphi}=\mathcal{J}(\varphi)=\{p \varphi: \quad p \in \mathbb{C}[z]\} .
$$

Clearly, the relations (3.9) hold for an invertible function $\varphi$. [6, Theorem 1] shows that they may also be valid when the function $\varphi$ is not invertible.

Let $\varphi \in \mathcal{P}(a ; b)$ be such a function that the submodule $\mathcal{J}(\varphi)$ contains at least one function $\Phi=\omega \varphi$, where $\omega$ is not a polynomial. Then, as it is proved in [6. Theorem 2], the principal submodule $\mathcal{J}_{\varphi}$ may be weakly localizable only if its generator $\varphi$ is an element of the space $\mathcal{F}\left(C_{0}^{\infty}(a ; b)\right)$. In the other hand, it follows from [4, Theorem 1.2] and [6, Theorem 3], the inclusion

$$
\varphi \in \mathcal{F}\left(C_{0}^{\infty}(a ; b)\right)
$$

is not a sufficient condition for the principal submodule $\mathcal{J}_{\varphi}$ to be weakly localizable.

To get any criteria of weak localizability for the principal submodule $\mathcal{J}_{\varphi}$ in terms of characteristics of its generator $\varphi$ seems to be quite difficult 
task. It can be reduced to the equivalent problem of weighted polynomial approximation. We are going to study it later in other place. Here we confine ourselves to considering an example of weakly localizable principal submodule generated by a function $\varphi \in \mathcal{F}\left(C_{0}^{\infty}(a ; b)\right)$.

Example. Suppose that $a<-\pi$ and $b>\pi$. Then, the functions

$$
\begin{array}{r}
s(z)=\frac{\sin \pi z}{\pi z}, \quad s_{1}(z)=s(\sqrt{z}), \\
\varphi(z)=\frac{s(z)}{s_{1}(z) s_{1}(-z)}
\end{array}
$$

belong to the module $\mathcal{P}(a ; b)$.

We assert that the principal submodule $\mathcal{J}_{\varphi} \subset \mathcal{P}(a ; b)$ is weakly localizable. It is well-known that

$$
\begin{array}{r}
|s(z)| \leq \frac{c_{0} e^{\pi|\operatorname{Im} z|}}{\pi(1+|z|)}, \quad z \in \mathbb{C}, \\
|s(z)| \geq \frac{m_{d} e^{\pi|\operatorname{Im} z|}}{\pi|z|}, \quad|z-k| \geq d, \quad k \in \mathbb{Z},
\end{array}
$$

where $c_{0}$ is an absolute constant, $d \in(0 ; 1 / 2)$, and $m_{d}$ is a positive constant depending on $d$. The estimates (3.13) and (3.14) imply that the relations (3.9) are valid for the submodules $\mathcal{J}_{s}$ and $\mathcal{J}(s)$. By Theorem 2 , the desired assertion will follow from the inclusion

$$
s \in \mathcal{J}_{\varphi}
$$

To prove (3.15) we should approximate the function $s$ in the space $\mathcal{P}(a ; b)$ by functions of the form $p \varphi$, where $p$ is a polynomial. Set $\omega(z)=s_{1}(z) s_{1}(-z)$. It follows from (3.13) that for any real $x$ the inequalities

$$
|\omega(x)| \leq \frac{c_{0}^{2} e^{\pi \sqrt{|x|}}}{\pi^{2}(1+\sqrt{|x|})^{2}} \leq c_{1} e^{\pi \sqrt{|x|}}
$$

hold, where $c_{1}=\frac{c_{0}^{2}}{\pi^{2}}$.

We are going to apply the following lemma (see [6, Lemma 1]) to get a proper estimate for the function $\varphi$ on the real axis. 
Lemma 2 Let $d_{0} \in(0 ; 1 / 2)$ be so small that $\left|\frac{\sin \pi \xi}{\pi \xi}-1\right| \leq 1 / 2$ for any $\xi \in \mathbb{C}$ satisfying $\pi|\xi| \leq d_{0}$. Then, there exists a positive constant $c_{d_{0}}$ depending on $d_{0}$ such that the inequality

$$
\left|s_{1}(z)\right| \geq \frac{c_{d_{0}} e^{\pi \sqrt{|z|} \sin (\theta / 2) \mid}}{1+|z|},
$$

holds if $z \in \mathbb{C} \backslash \bigcup_{k \in \mathbb{N}}\left\{z:\left|z-k^{2}\right|<3 d_{0}\right\}$.

From the relations (3.12), (3.13), (3.17), we derive the estimates for the function $\varphi$ :

$$
\begin{aligned}
& |\varphi(z)| \leq \frac{c_{0}(1+|z|)}{\pi c_{d_{0}}^{2} e^{\pi \sqrt{|z|}}} \\
& \text { if } z \in \mathbb{R} \backslash \bigcup_{k \in \mathbb{N}}\left(\left\{z:\left|z-k^{2}\right|<3 d_{0}\right\} \bigcup\left\{z:\left|z+k^{2}\right|<3 d_{0}\right\}\right) \\
& |\varphi(z)| \leq \frac{c_{0} e^{3 \pi d_{0}}(1+|z|)}{c_{d_{0}}^{2} \pi e^{\pi \sqrt{|z|}(|\sin (\theta / 2)|+|\cos (\theta / 2)|)}} \\
& \text { if } z \in \bigcup_{k \in \mathbb{N}}\left(\left\{z:\left|z-k^{2}\right|=3 d_{0}\right\} \bigcup\left\{z:\left|z+k^{2}\right|=3 d_{0}\right\}\right) .
\end{aligned}
$$

Applying the maximum modulus principle, we get

$$
|\varphi(x)| \leq C_{1}(1+|x|) e^{-\pi \sqrt{|x|}}
$$

for any $x \in \mathbb{R}$, where $C_{1}$ depends only on $d_{0} \in(0 ; 1 / 12)$.

We set $W(x)=(1+|x|) e^{\pi \sqrt{|x|}}$ to be a weight function. By the theorem due to de Branges (see [18, VI.H.1]) and the second theorem in the paragraph VI.H.2 of the same book, we deduce that there exists a sequence of polynomials $p_{m}$ satisfying the relation

$$
\left\|p_{m}-\omega\right\|_{W}=\sup _{x \in \mathbb{R}} \frac{\left|p_{m}(x)-\omega(x)\right|}{W(x)} \rightarrow 0 \quad \text { as } \quad m \rightarrow+\infty .
$$

Taking into account the inequality (3.18), we obtain that

$$
\left|p_{m}(x) \varphi(x)\right| \leq C(1+|x|)^{2}, \quad x \in \mathbb{R}, \quad m=1,2, \ldots
$$


These estimates, together with the Phragmen-Lindelöf principle and the topological properties of the space $\mathcal{P}(a ; b)$, lead to the boundedness of the sequence $p_{m} \varphi$ in $\mathcal{P}(a ; b)$. By [10, Corollary 3], we conclude that there is a subsequence $p_{m_{j}} \varphi$, which converges to the function $s$ in the space $\mathcal{P}(a ; b)$.

In the following theorem we obtain sufficient conditions of weak localizability for a submodule $\mathcal{J} \subset \mathcal{P}(a ; b)$ without a priori requirement of its stability.

Theorem 3 Let $\mathcal{J} \subset \mathcal{P}(a ; b)$ be a closed subspace. Assume that the intervals $(a ; b),\left(c_{\mathcal{J}} ; d_{\mathcal{J}}\right)$ are unbounded, and for any $\varphi \in \mathcal{J}$ the implication

$$
e^{i h z} \varphi \in \mathcal{P}(a ; b), \quad h \in \mathbb{R} \Longrightarrow e^{i h z} \varphi \in \mathcal{J}
$$

is true. Then, $\mathcal{J}$ is a weakly localizable submodule.

Proof. Without loss of generality, we suppose that

$$
a=0, \quad b=d_{\mathcal{J}}=+\infty .
$$

Let us notice that

$$
\frac{e^{\mathrm{i} \tau z} \varphi-\varphi}{\tau} \rightarrow z \varphi \quad \text { as } \quad \tau \rightarrow 0
$$

in the topology of $\mathcal{P}(a ; b)$. Together with the implication (3.19), it leads to the conclusion that $\mathcal{J}$ is a submodule. By Corollary 1, we need only to prove the stability property for this submodule .

It is known that the stability of a (closed) submodule at any point $\lambda \in \mathbb{C}$ follows from its stability at one point $\lambda_{0}$ (see [15, Proposition 4.2, remark 1, $\S 4])$. Let us fix a point $\lambda_{0} \in \mathbb{C} \backslash \Lambda_{\mathcal{J}}$ and show that $\frac{\psi}{z-\lambda_{0}} \in \mathcal{J}$ for any function $\psi \in \mathcal{J}$ vanishing at the point $\lambda_{0}$. According to the definition of the zero set $\Lambda_{\mathcal{J}}$, we can find a function $\varphi_{0} \in \mathcal{J}$ with the property $\varphi_{0}\left(\lambda_{0}\right)=1$.

Let $S=\mathcal{F}^{-1}(\psi), S_{\lambda_{0}}=\mathcal{F}^{-1}\left(\frac{\psi}{z-\lambda_{0}}\right), S_{0}=\mathcal{F}^{-1}\left(\varphi_{0}\right), \tilde{S}_{0}=\mathcal{F}^{-1}\left(\frac{\varphi_{0}-1}{z-\lambda_{0}}\right)$.

Then, for the distributions

$$
S_{1}=\mathcal{F}^{-1}\left(\frac{\varphi_{0}-1}{z-\lambda_{0}} \psi\right), \quad S_{2}=\mathcal{F}^{-1}\left(\frac{\psi}{z-\lambda_{0}} \varphi_{0}\right)
$$

we have

$$
\left(S_{1}, f\right)=\left(\tilde{S}_{0}, S * f\right), \quad\left(S_{2}, g\right)=\left(S_{\lambda_{0}}, S_{0} * f\right)
$$


where $(S * f)(\tau)=(S, f(t+\tau)), f \in \mathcal{E}(0 ;+\infty)$.

Let $W \subset \mathcal{E}(0 ;+\infty)$ be the subspace which annihilator submodule is $\mathcal{J}$. Then, (3.19) and (3.20) imply that the relations

$$
\left(S_{1}, f\right)=0, \quad\left(S_{2}, f\right)=0, \quad \text { for any } \quad f \in W .
$$

In what follows, $S_{1}, S_{2} \in W^{0}$ or, equivalently,

$$
\frac{\varphi_{0}-1}{z-\lambda_{0}} \psi \in \mathcal{J}, \quad \frac{\psi}{z-\lambda_{0}} \varphi_{0} \in \mathcal{J} .
$$

From the last relations we conclude that

$$
\frac{\psi}{z-\lambda_{0}}=\frac{\psi}{z-\lambda_{0}} \varphi_{0}-\frac{\varphi_{0}-1}{z-\lambda_{0}} \varphi_{0} \in \mathcal{J} .
$$

\section{Dual assertions on $D$-invariant subspaces}

In this section we obtain the assertions on closed subspaces $W \subset \mathcal{E}(a ; b)$, which are equivalent to the theorems proved in the previous one.

From here and thereon, unless otherwise specified, we denote by $W$ a closed $D$-invariant subspace of $\mathcal{E}(a ; b)$ with discrete spectrum $\sigma_{W}=-\mathrm{i} \Lambda$, where $\Lambda=\left\{\left(\lambda_{j} ; m_{j}\right)\right\}$.

Theorem 4 For the subspace $W$ the representation

$$
W=\overline{W_{I_{W}}+\mathcal{L}(\operatorname{Exp} W)}
$$

holds (in the other words, $W$ admits weak spectral synthesis) if and only if its annihilator submodule $\mathcal{J}$ contains a function $\varphi$ with the property

$$
\mathcal{J}(\varphi) \subset \mathcal{J}
$$

Proof. By [3, Proposition 3.1], the annihilator submodule $\mathcal{J}=\mathcal{F}\left(W^{0}\right)$ is stable. Proposition 2 means that the representation (4.1) holds if and only if the submodule $\mathcal{J}$ is weakly localizable. In what follows, Theorem 1 leads to the required assertion.

The following theorem is dual to Theorem 2 . 
Theorem 5 Let the subspace $W$ satisfy one of the assumptions listed below.

1) The annihilator submodule $\mathcal{J}=\mathcal{F}\left(W^{0}\right)$ contains a function $\varphi$ generating weakly localizable principal submodule $\mathcal{J}_{\varphi}$.

Or,

2) the completeness radius $\rho_{\Lambda}$ is less than a half of the length of the interval $I_{W}$.

Then $W$ admits weak spectral synthesis.

Corollary 2 The subspace $W$ admits classical spectral synthesis (1.1) if and only if $I_{W}=(a ; b)$.

For any $A, B \subset \mathbb{R}$ we denote by $A \div B$ their geometric difference, which is equal to the set of all $x \in \mathbb{R}$ such that $x+B \subset A$. Let $S \in \mathcal{E}^{\prime}(a ; b)$ and $h \in(a ; b) \div \operatorname{ch} \operatorname{supp} S$ (by ch supp $S$ we denote the convex hall of $\operatorname{supp} S)$. We define $h$-translation $S_{h}$ setting $\left(S_{h}, f\right)=(S, f(t+h))$ for any $f \in \mathcal{E}(a ; b)$.

Theorem 6 Let $W \subset \mathcal{E}(a ; b)$ be an arbitrary closed subspace, and let both intervals $(a ; b), I_{W}$ be unbounded. Assume that the annihilator subspace $W^{0}$ is invariant with respect to the translation operator, that is, the inclusion $S \in W^{0}$ implies that $S_{h} \in W^{0}$ for any $h \in(a ; b) \div \operatorname{ch} \operatorname{supp} S$.

Then, the subspace $W$ is D-invariant and admits weak spectral synthesis.

Proof. The assumption on the annihilator subspace $W^{0}$ implies that the subspace $W$ is $D$-invariant, and the annihilator submodule $\mathcal{J}=\mathcal{F}\left(W^{0}\right)$ satisfies the implication (3.19). By Theorem 3, this submodule is weakly localizable. Therefore, the subspace $W$ admits weak spectral synthesis (Proposition 2).

\section{References}

[1] L. Euler De integratione aequationum differentialum altiorum gradum.// Miscellanea Berol. 1743. No. 7. Pp. 193-242.

[2] L. Schwartz “Theorie générale des fonctions moyenne-périodique Ann. of Math. 1947. V. 48. No. 4. Pp. 857-929. 
[3] A. Aleman, B. Korenblum. Derivation-Invariant Subspaces of $C^{\infty} . / /$ Computation Methods and Function Theory. 2008. V. 8. No. 2. Pp. 493-512.

[4] A. Aleman, A. Baranov, Yu. Belov. Subspaces of $C^{\infty}$ invariant under the differentiation.// arXiv:1309.6968v2 [math.CV]

[5] A. Beurling, P. Malliavin. On the closure of characters and the zeros of entire functions.// Acta Math. 1967. V. 118. No. 1-4. Pp. 79-93.

[6] N.F. Abuzyarova. Some properties of principal submodules in the module of entire functions of exponential type and polynomial growth on the real axis.// Ufa Math. J. 2016. V. 8. No. 1 (to appear).

[7] N.F. Abuzyarova. Spectral synthesis in the Schwartz space of infinitely differentiable functions.// Doklady Mathematics. 2014. V. 90. No. 1. Pp. 479-482.

[8] I.F. Krasichkov-Ternovskii. Invariant subspaces of analytic functions. I. Spectral analysis on convex regions.// Matem. Sbornik. 1972. V.87(129). No. 4. Pp. 459-489 [Math. USSR-Sbornik. 16:4, 471-500 (1972).]

[9] L. Ehrenpreis. Mean periodic functions. Amer. J. Math. 1955. V. 77. No. 2. Pp. $293-326$.

[10] J. Sebastian-e-Silva. On some classes of locally convex spaces important in applications. // Matematika. Sbornik Perevodov. 1957. 1:1. Pp. 60-77 (in Russian).

[11] L. Hörmander. The analysis of linear partial differential operators I: distribution theory and Fourier analysis. Springer. Berlin. 1990.

[12] B. Y. Levin (in collaboration with Yu. Lyubarskii, M. Sodin, V. Tkachenko). Lectures on entire functions (Rev. Edition). AMS. Providence. Rhode Island, 1996. 254 p.

[13] N.F. Abuzyarova. Closed submodules in the module of entire functions of exponential type and polynomial growth on the real axis. Ufa Math. J. 2014. V. 6. No. 4. Pp. 3-17. 
[14] I.F. Krasichkov-Ternovskii. Local description of closed ideals and submodules of analytic functions of one variable. I // Izvestia AN SSSR. Ser. Matem. 1979. V. 43. No.1. Pp. 44-66. [Math. USSR-Izvestiya. 14:1, 41-60 (1980).]

[15] I.F. Krasichkov-Ternovskii. Local description of closed ideals and submodules of analytic functions of one variable. II // Izvestia AN SSSR. Ser. Matem. 1979. V. 43. No. 2. Pp. 309-341. [Math. USSRIzvestiya. 14:2, 289-316 (1980).]

[16] I.F. Krasichkov-Ternovskii. Invariant subspaces of analytic functions. III. On the extension of spectral synthesis.// Matem. Sbornik. 1972. V.88(130). No. 3. Pp. 331-352. [Math. USSR-Sbornik. 17:3, 327-348 (1972).]

[17] C.A. Berenstein, B.A. Taylor. A new look at interpolation theory for entire functions of one variable.// Advances in Mathematics. 1980. V. 33. P. 109-143.

[18] P. Koosis. The logarithmic integral I. Cambridge Univ. Press. 1998. 606 pp.

Natalia Fairbakhovna Abuzyarova, Baskir State University, Zaki Validi str., 32 450074, Ufa, Russia abnatf@gmail.com 